\title{
A case of adalimumab-induced pneumonitis in a 45-year-old man with Crohn's disease
}

\author{
James D Reid MD, Brian Bressler MD MS FRCPC, John English MD FRCPC
}

\author{
JD Reid, B Bressler, J English. A case of adalimumab-induced \\ pneumonitis in a 45-year-old man with Crohn's disease. Can \\ Respir J 2011;18(5):262-264.
}

\begin{abstract}
Adalimumab is a human monoclonal antibody against tumour necrosis factor-alpha that has been associated with acute lung toxicity, mainly in patients with rheumatoid arthritis. Descriptions of similar patterns of lung injury in patients treated with adalimumab for inflammatory bowel disease are emerging in the literature. A case involving a 45-year-old man with Crohn's disease who developed a nonbronchiolitis inflammatory nodular pattern of lung injury after starting adalimumab is reported.
\end{abstract}

Key Words: Acute drug reaction; Adalimumab; Antitumour necrosis factoralpha; Drug-induced lung disease; Drug-induced lung toxicity; Interstitial lung disease

\begin{abstract}
dalimumab is a monoclonal antibody against tumour necrosis A factor-alpha (TNF $\alpha)$ that is used to treat refractory Crohn's disease. Anti-TNF medications have been associated with a variety of lung pathologies (1-3). Making a diagnosis of anti-TNF $\alpha$-related lung injury in patients with Crohn's disease is complicated by potential additional lung injury patterns contributed by methotrexate $(3,4)$ and underlying inflammatory bowel disease (IBD) (5). We present a case involving a 45-year-old man who developed acute pulmonary toxicity after starting adalimumab for Crohn's disease. The lung biopsy showed a nonbronchiolitis inflammatory nodular pattern. These findings would be atypical for pulmonary involvement of the patient's IBD (5). An extensive infectious workup was negative. The literature contains several cases of anti-TNF $\alpha$-induced interstitial lung disease in patients with Crohn's disease, each with a unique pathological appearance $(2,6,7)$. The clinical and pathological picture in the present case supports an adalimumab-induced pulmonary toxicity.
\end{abstract}

\section{CASE PRESENTATION}

A 45-year-old man was diagnosed with Crohn's disease in January 2008 after presenting with six weeks of diarrhea, arthralgias and $11.8 \mathrm{~kg}$ of weight loss. After multiple attempts, his disease was not controlled on steroid-sparing agents. In July 2008, he was started on methotrexate; however, his symptoms did not improve and, in October, he was started on adalimumab.

In August 2009, the patient developed fever, chills, diaphoresis, nonproductive cough, exertional dyspnea and bloody diarrhea. His medical history included a 30 pack-year smoking history.

On physical examination, his temperature was $37.8^{\circ} \mathrm{C}$, he was not hypoxic and the remainder of his vital signs were normal. There were no auscultative or percussive findings to suggest pneumonia. His white blood cell count was $13.2 \times 10^{9} / \mathrm{L}$.

A computed tomography scan of the chest showed subpleural reticulations and ground glass opacities, consistent with nonspecific interstitial pneumonia, along with diffuse areas of tree-in-bud nodularity suggesting superimposed cellular bronchiolitis (Figure 1).

The patient received intravenous antibiotics for presumptive bacterial pneumonia, which did not help. Extensive bacterial, viral and

\section{Un cas de pneumonite induite par l'adalimumab chez un homme de 45 ans atteint de la maladie de Crohn}

L'adalimumab est un anticorps monoclonal humain contre le facteur de nécrose tumorale alpha qui s'associe à une toxicité pulmonaire aiguë, surtout chez les patients atteints de polyarthrite rhumatoïde. Des descriptions de profils de lésions pulmonaires similaires chez des patients prenant de l'adalimumab pour traiter une maladie inflammatoire de l'intestin émergent dans les publications. Est exposé le cas d'un homme de 45 ans atteint de la maladie de Crohn qui a présenté un profil inflammatoire de lésion pulmonaire nodulaire non bronchiolitique après avoir entrepris un traitement à l'adalimumab.

fungal cultures, stains and serology including those for tuberculosis and histoplasmosis were negative.

A wedge biopsy of the right upper lobe demonstrated multiple whitish nodules measuring $0.2 \mathrm{~cm}$ to $0.3 \mathrm{~cm}$ in size on gross section (Figure 2). On microscopy, these corresponded to a series of individual and confluent nodules composed of an intimate mixture of lymphocytes and histiocytes. In the larger nodules, a progressively increased number of neutrophils could be seen to the point in which they could be considered microabscesses. The periphery of the nodules showed prominent airspace fibrin, which was also incorporated within the nodular inflammatory infiltrate (Figure 3 ). Less developed inflammatory nodules could be described as vaguely granulomatous; however, multinucleated histiocytic giant cells were not observed. Regions of intervening lung parenchyma were relatively unaffected. The nodular lesions were not believed to be centred on bronchioles. Multiple blocks were examined for bacteria, acid-fast bacilli and fungi and all were negative for microorganisms. A drug-related lung injury was suspected and the adalimumab and methotrexate were discontinued in August 2009

The patient's dyspnea responded to pulse steroids, and he showed slow but ongoing clinical and radiographic improvement on prednisone. Methotrexate was reinitiated in October 2009 without any subsequent compromise in the patient's respiratory status.

\section{DISCUSSION}

Descriptions of lung disease attributed to anti-TNFa vary considerably, ranging from interstitial pneumonitis to malignancy (1). Ramos-Casals et al (2) reported 18 cases of interstitial lung disease in 226 patients with autoimmune diseases secondary to TNF-targeted therapies. The majority of these patients had rheumatoid arthritis, while only two had Crohn's disease. One-half of these patients were treated concomitantly with methotrexate, and the authors postulated a methotrexate-potentiating effect through deficient apoptosis of infiltrating inflammatory cells. Weatherhead et al (6) described a case of severe interstitial pneumonitis secondary to infliximab infusions in a 22-year-old woman with fistulating Crohn's disease. Unlike the present case, their patient experienced complete symptom resolution after a

${ }^{1}$ Department of Medicine; ${ }^{2}$ Division of Gastroenterology, St Paul's Hospital; ${ }^{3}$ Department of Pathology Eु Laboratory Medicine, University of

British Columbia, Vancouver, British Columbia

Correspondence: Dr James D Reid, Department of Medicine, University of British Columbia, 304-457 West Avenue, Kelouna, British Columbia V1Y $4 Z 3$.

Telephone 250-212-8546, fax 250-368-0054, e-mail jdreid@interchange.ubc.ca 


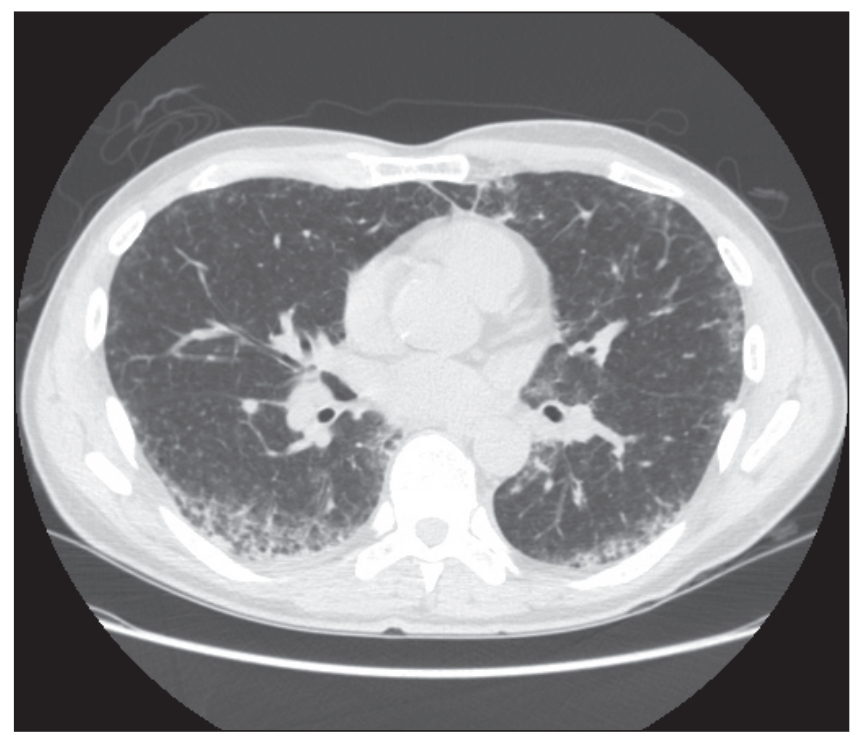

Figure 1) Computed tomography scan showing subpleural reticulations, ground-glass opacities and diffuse areas of tree-in-bud nodularity

two-week course of steroids. Furthermore, there were no inflammatory changes seen on bronchial biopsy. Weine et al (7) described a case of lupus-like acute pulmonary inflammation in a 51 -year-old woman on infliximab for ulcerative colitis. It was believed that the lung disease was secondary to drug-induced obliterative bronchiolitis. Gradual resolution of the dyspnea was seen after discontinuing infliximab. Similar to the present case, a mixed interstitial inflammatory cell infiltrate was seen on pathology, but there was no description of nodular or granulomatous findings.

A recent report by Yousem and Dacic (8) described four patients with non-necrotizing interstitial and airspace lymphohistiocytic granulomas following etanercept therapy for rheumatoid arthritis. The authors noted a prominent lymphocytic component within the granulomas and an acute fibrinous component to the pneumonia. Our case was also notable for a fibrinous exudate and dense lymphocytichistiocytic admixture as well as the somewhat novel manifestation of microabscess formation.

The literature on adverse effects of anti-TNF $\alpha$ antibody therapy typically divides complications into infectious and noninfectious categories. The infectious causes include granulomatous (mycobacterial and fungal/pneumocystis agents) and nongranulomatous manifestations (bacterial infections) $(3,9,10)$. Diffuse lung disease, believed solely to be due to anti-TNF $\alpha$ therapy, has been described, although many of these examples are in the form of case reports, and the nature of the lung disease is often based on radiological imaging without benefit of video-assisted thorascopic surgery or open-lung wedge biopsy sampling. Nevertheless, a pattern of non-necrotizing granulomatous pneumonitis is frequently found on pathological examination $(9,10)$. Assignation of this injury to anti-TNF $\alpha$ agents is often presumptive, and acceptable only after ruling out infection and contributions from underlying diseases of concomitant therapies. Methotrexate, for example, is well recognized to produce a non-necrotizing granulomatous pneumonitis as part of the pathological spectrum of lung injury (4).

A broad differential of potential causes of pneumonitis were considered in the presented case. Methotrexate seems unlikely to be the etiology of lung disease in this case because the patient tolerated the reintroduction of this medication. Furthermore, methotrexate pneumonitis typically presents within 32 weeks of initiating the drug (3). In the present case, methotrexate had been given for one full year before the onset of pulmonary complications. Infection could not be completely excluded; however, all cultures and serological markers were negative. Furthermore, there was no improvement with antibiotic

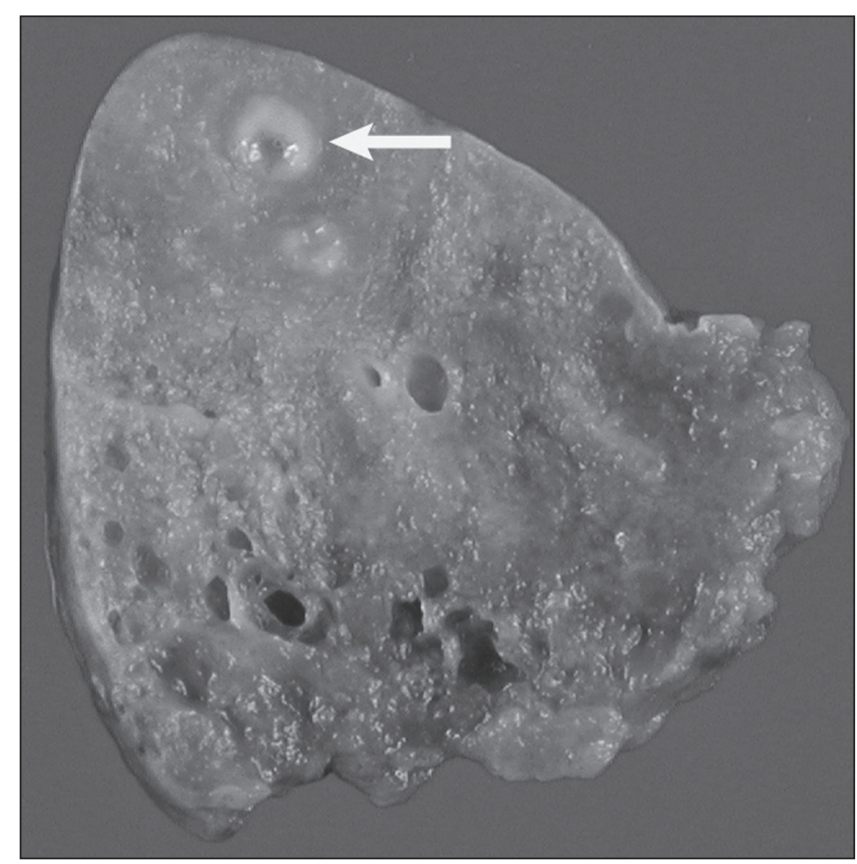

Figure 2) Gross specimen of lung wedge biopsy demonstrating multiple whitish nodules. The white arrow marks an individual nodule

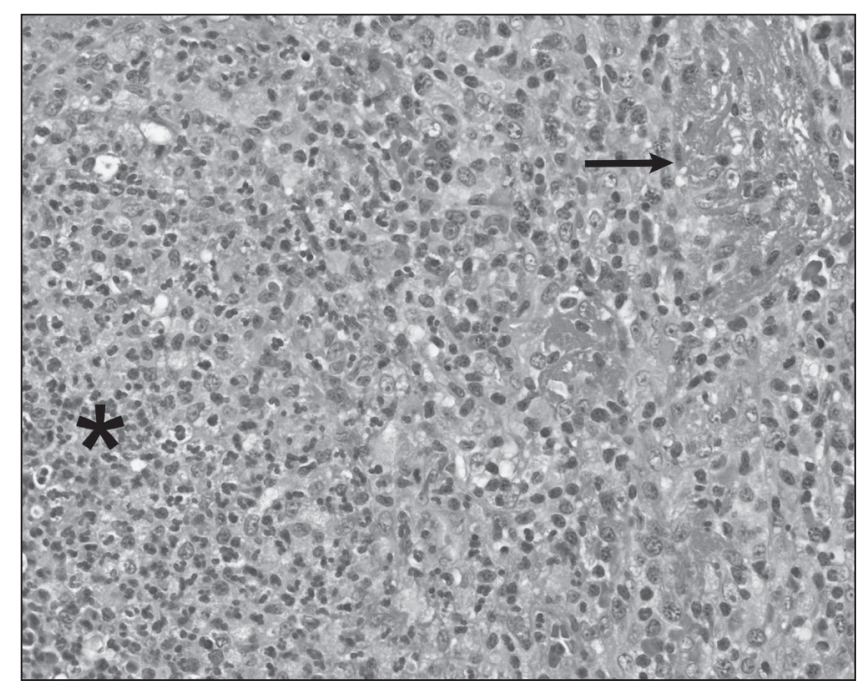

Figure 3) Microscopic view of lung biopsy focusing on a nodule. Asterisk marks a larger nodule, demonstrating a progressively increased number of neutrophils, resembling a microabscess. The black arrow marks prominent airspace fibrin in the periphery of the nodule

therapy and, eventually, a response was seen with steroids. The acute cellular (neutrophilic) bronchiolitis component of the pathology in our case could be consistent with the broad pathological spectrum of inflammatory bowel disease-related lung injury (5). However, there was no evidence of chronic (fibrous) obliterative bronchiolitis which would be more suggestive of IBD-related lung disease (5). Although the smaller conducting airways contained organizing luminal exudate, it was believed that the inflammatory process likely had its epicentre in the peribronchiolar acini, with extrusion of cells and fibrin more proximally. When membranous bronchioles were seen isolated within the parenchyma, no intrinsic bronchiolitis was observed.

Attributing lung disease to adalimumab in the present case was based on the temporal association between inception of therapy, onset of respiratory symptoms, a negative infectious workup, the 
pathological appearance on biopsy, and the clinical improvement following discontinuation of the adalimumab and subsequent reintroduction of methotrexate. Although rare, anti-TNF $\alpha$-induced lung disease is a potentially harmful side effect of this medication, which should be further investigated to determine the mechanism of injury and predisposing patient characteristics.

\section{REFERENCES}

1. Takao K, Masayoshi H, Shigeko I, et al. Postmarketing surveillance of the safety and effectiveness of etanercept in Japan. J Rheumatol 2009;36:898-906.

2. Ramos-Casals M, Brito-Zeron P, Munoz S, et al. Autoimmune diseases induced by TNF-targeted therapies: Analysis of 233 cases. Medicine 2007;86:242-51.

3. Thavarajah K, Wu P, Rhew E, Yeldani A, Kamp D. Pulmonary complications of tumor necrosis factor-related therapy. Respir Med 2009;103:661-9.
4. Imokawa S, Colby T, Leslie KO, Helmers R. Methotrexate pneumonitis: Review of the literature and histopathological findings in nine patients. Eur Respir J 2000;15:373-81.

5. Casey M, Tazelaar H, Myers J, et al. Noninfectious lung pathology in patients with Crohn's disease. Am J Surg Pathol 2003;27:213-9.

6. Weatherhead M, Masson S, Bourke S, Gunn M, Burns G. Interstitial pneumonitis after infliximab therapy for Crohn's disease - a case. Inflamm Bowel Dis 2006;12:427-8.

7. Weine D, Schamberg N, Maltz C. Infliximab induced lung disease. American College of Gastroenterology Annual Scientific Meeting and Postgraduate Course, 2009. < http://acgconnect.bdmetrics.com/ SOW-2855615/Abstract-P1145-Infliximab-Induced-Lung-Disease/ Overview.aspx > (Accessed on January 1, 2010). (Abst)

8. Yousem S, Dacic S. Pulmonary lymphohistiocytic reactions temporally related to etanercept therapy. Mod Pathol 2005;18:651-5.

9. Dine C, Kreider M. Pulmonary complications of antirheumatic therapies. Clin Pulmon Med 2009;16:315-22.

10. Mutlu G, Mutlu E, Bellmeyer A, Rubinstein I. Pulmonary adverse events of anti-tumor necrosis factor- $\alpha$ antibody therapy. Am J Med 2006;119:639-46. 


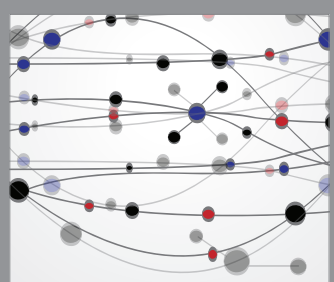

The Scientific World Journal
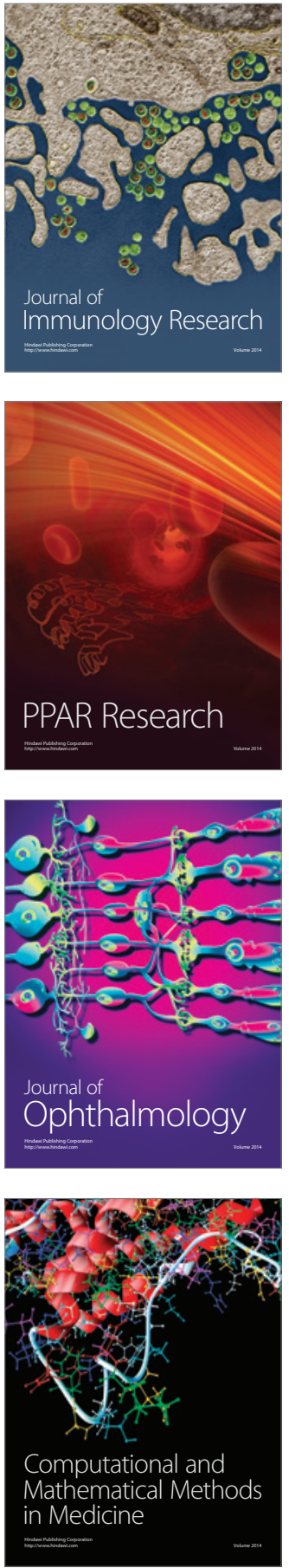

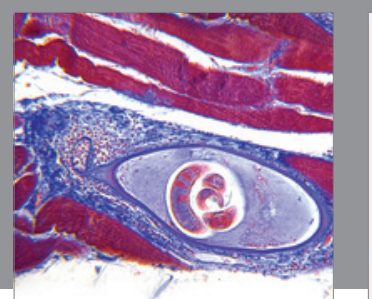

Gastroenterology Research and Practice

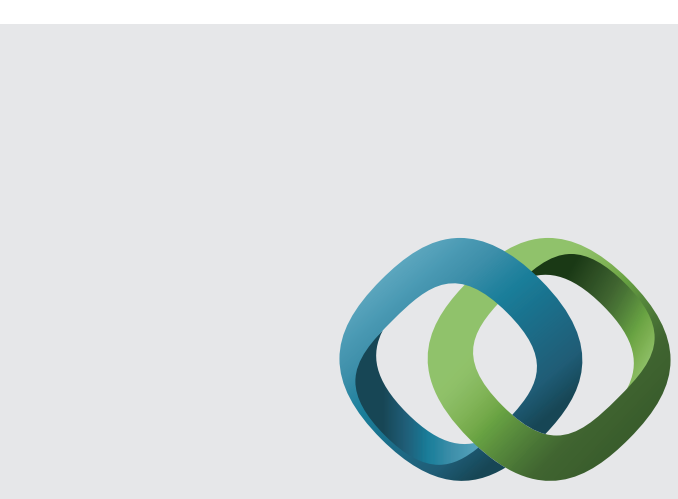

\section{Hindawi}

Submit your manuscripts at

http://www.hindawi.com
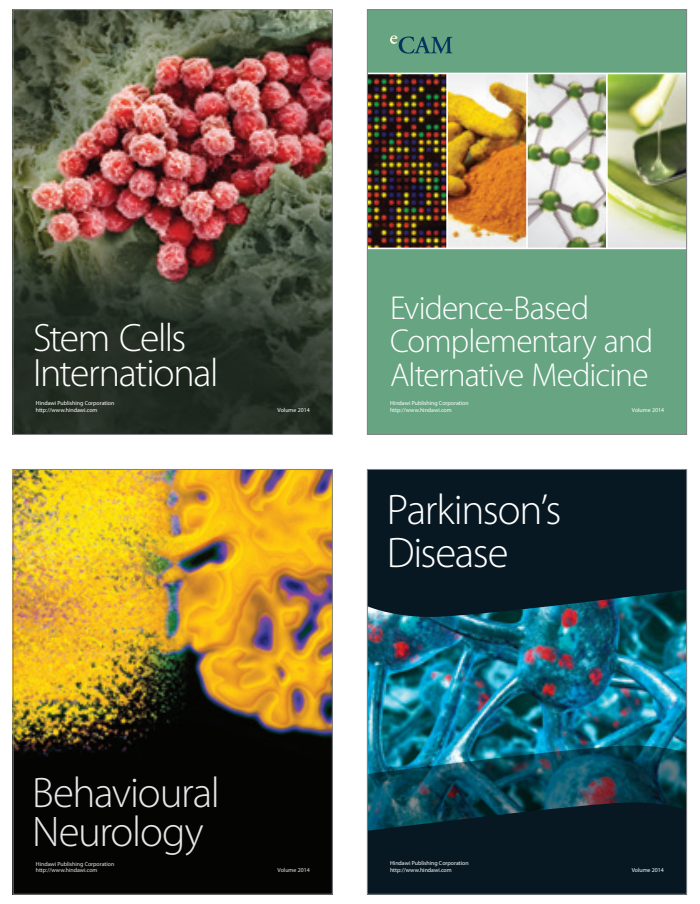
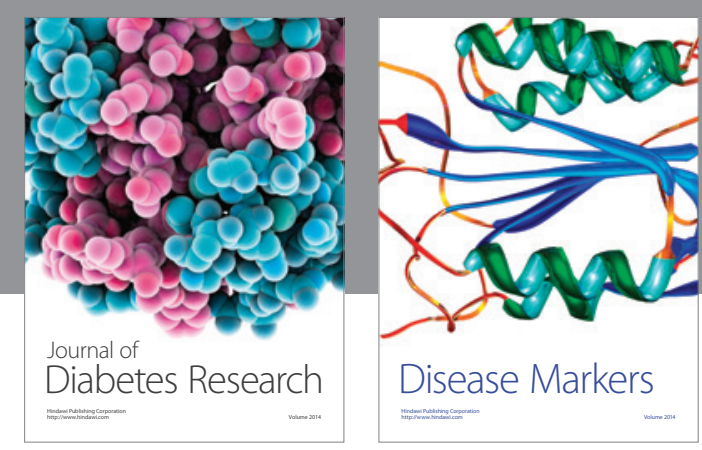

Disease Markers
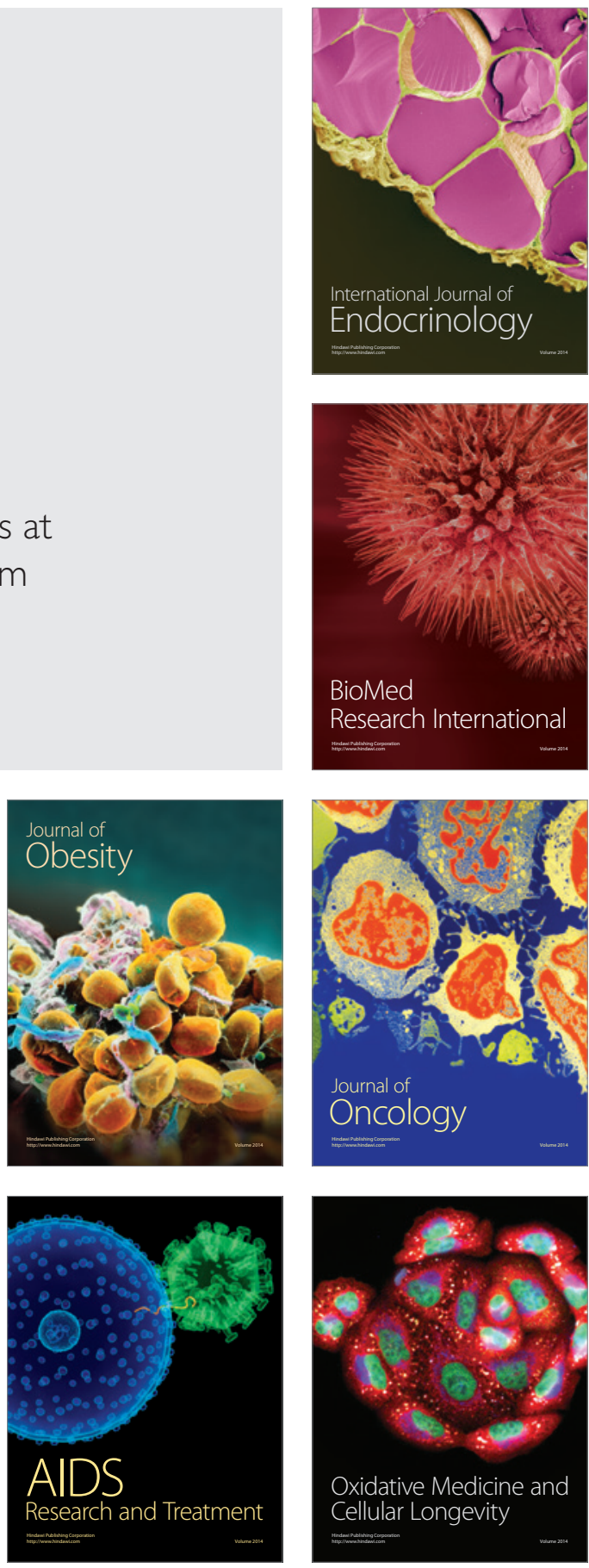